\title{
VALORES DE REFERENCIA DEL ÍNDICE DE PULSATILIDAD DE LA ARTERIA CEREBRAL MEDIA EN FETOS SANOS ENTRE LAS 20 A 40 SEMANAS EN LIMA- PERÚ
}

\author{
Oscar Antonio Limay Rios ${ }^{1}$, Roxana Guadalupe Calixto Coronel ${ }^{2}$, Jaime Ingar Pinedo ${ }^{3}$, \\ Erasmo Huertas Tachinno ${ }^{4}$, Walter Castillo Urquiaga ${ }^{4}$, Walter Ventura Laveriano ${ }^{4}$, Mario Zarate Girao ${ }^{4}$.
}

\begin{abstract}
RESUMEN
Objetivo: Establecer valores de referencia del índice de pulsatilidad de la arteria cerebral media en fetos sanos. Método: Es un estudio descriptivo, retrospectivo y transversal realizado en el Instituto Nacional Materno Perinatal en el periodo 2014 al 2016.Se registraron 1865 mujeres con embarazo único y bajo riesgo entre las 20 a 40 semanas de gestación y se le realizaron examen Doppler de la arteria cerebral media fetal. Resultados: La edad promedio de las gestantes fue de 29,23 años y distribución porcentual según el grupo etario y de mayor frecuencia fueron las gestantes de 20 a 34 años con $66,2 \%$, seguida de las gestantes añosas con $24,9 \%$ y por ultimo las gestantes de 13 a19 años con un $8,9 \%$.El índice de pulsatilidad de la arteria cerebral media mostró aumento inicial hasta alcanzar un pico máximo a las 28 semana de gestación y disminuyó posteriormente hasta terminar la gestación. El valor del IP en el percentil 5 fue 1.28, del percentil 50 fue 1.99 y por ultimo del percentil 95 fue 3.11 . La curva normal del IP de ACM de población en estudio siguió un patrón parabólico. Conclusión: Se establecieron valores de referencia del índice de pulsatilidad de la arteria cerebral media en el percentil 5,50 y 95.Los valores del índice de pulsatilidad de la ACM entre el percentil 5 y 95 demuestra tener un valor clínico en la detección de fetos en condiciones óptimas.
\end{abstract}

Palabras clave: Arteria cerebral media; Indice de pulsatilidad; Valores de referencia; Ultrasonografía doppler (Fuente DeCS BIREME)

\section{REFERENCE VALUES OF THE PULSATILITY INDEX OF THE MEDIUM BRAIN ARTERY IN HEALTHY FETUS BETWEEN 20 TO 40 WEEKS IN LIMA-PERÚ}

\begin{abstract}
Objective: To establish reference values of pulsatility index of the middle cerebral artery in healthy fetuses. Method: This is a descriptive, retrospective and cross-sectional study conducted at the National Maternal Perinatal Institute between 2014 and 2016. 1865 women with a single pregnancy and low risk were registered between 20 and 40 weeks of gestation and were screened Doppler Fetal middle cerebral artery. Results: The average age of pregnant women was 29.23 years and percentage distribution according to the age group and the highest frequency were pregnant women aged 20 to 34 years old with $66.2 \%$, followed by the aged pregnant women with $24.9 \%$ and finally the pregnant women from 13 to 19 years old with $8.9 \%$. The pulsatility index of the middle cerebral artery showed an initial increase until reaching a maximum peak at 28 weeks of gestation and subsequently decreased until the end of gestation. The IP value in the 5 th percentile was 1.28 of the 50 th percentile 1.99 and last of the 95 th percentile was 3.11 . The normal ACM IP curve of the population under study followed a parabolic pattern. Conclusion: Reference values of the middle cerebral artery pulsatility index were established in the 5,50 and 95th percentile. The values of the pulsatility index of the ACM between the 5th and 95th percentile are shown to have a clinical value in the detection of fetuses in optimum conditions.
\end{abstract}

Key words: Middle cerebral artery; Pulsatility index; Reference values; Doppler ultrasonography (Source: MeSH NLM).

\section{INTRODUCCIÓN}

El ultrasonido Doppler es un instrumento no invasivo que nos permite el estudio de los procesos hemodinámicos del feto y de la placenta; asimismo nos permite conocer el bienestar fetal. Los cambios del flujo sanguíneo patológicos de los vasos principales como la arteria cerebral media son producto de un estado de hipoxia crónica que sufren los fetos a consecuencia de patologías maternas como la preeclampsia y retardo de crecimiento intrauterino relacionado frecuentemente a la insuficiencia placentaria ${ }^{1,2}$

La alteración de la flujometría doppler fetal se caracteriza por un aumento de la resistencia de la arteria umbilical y de manera inversa la arteria cerebral media, produciendo un fenómeno conocido como brain sparing; que se caracteriza por presentar redistribuir flujo sanguíneo a los órganos principales como el cerebro, el corazón y las glándulas suprarrenales; en el cerebro se representa por una vasodilación cerebral y ello indica que el IP de la ACM se encuentra por debajo del percentil cinco $^{3,4}$.

Existen diversos estudios internacionales que han establecido rangos de referencia del índice de pulsatilidad de la arteria cerebral media entre las semana 20 y 40 , en que se evidencia información variada, así como el estudio realizado por Baschat en Alemania señala un IP de ACM en el percentil cinco de 1.45, y Tavares en Brasil indica un

\footnotetext{
Médico Gineco-Obstetra Asistente del Servicio de Medicina Fetal. Jefe de Unidad de Investigación del Instituto Nacional Materno Perinatal. Lima-Perú

Estudiante de Facultad de Medicina Humana de la Universidad San Juan Bautista.Lima-Perú.

Médico Gineco-Obstetra Jefe del Servicio de Medicina Fetal del Instituto Nacional Materno Perinatal. Lima-Perú.

Médico Gineco-Obstetra Asistente del Servicio de Medicina Fetal del Instituto Nacional Materno Perinatal. Lima-Perú
}

Recibido: 01-12-16 Aprobado: 30-06-17 
valor de 1.74 en la semana 28 de gestación. En el Perú hasta la fecha no existen estudios que hayan establecido parámetros de referencias del IP de la ACM; es por ello que se ve la necesidad de crear tablas de referencia que reflejen la idiosincrasia de nuestra población ${ }^{5}$.

El estudio busca establecer valores de referencia del índice de pulsatilidad de la arteria cerebral media en el percentil 5,50 y 95 en fetos sanos de gestantes de 20 a 40 semanas de gestación atendidas en Instituto Nacional Materno Perinatal.

\section{MATERIALES Y MÉTODOS}

Tipo de estudio: Descriptivo, retrospectivo y transversal. La población de estudio: Todos Fetos sanos de Gestantes de 20 a 40 semanas que fueron atendidas en servicio de medicina fetal del Instituto Materno Perinatal en el periodo comprendido 2014 a 2016, a quienes se realizó el ultrasonido doppler.

Criterios de inclusión: Gestantes de 20 a 40 semanas confirmados por fecha de última regla y/o primera ecografía del primer trimestre, con gestación única y sin malformaciones fetales, y a las que se le midió el IP de la arteria cerebral media fetal entre las 20 y 40 semanas de gestación y que tuvieron un recién nacido sano al término de su embarazo.

Criterios de exclusión: Gestantes con datos incompletos en la historia clínica y con enfermedades que comprometan el estado general de la gestante.

La recolección de datos se realizó de dos fuentes: mediante la revisión de historias clínicas y la base de datos de ecografías de la Unidad de Medicina Fetal. En dichas fuentes consignaba nuestras variables a estudiar como el índice de pulsatilidad de la arteria cerebral media, edad gestacional al momento del Doppler y la edad materna.

En el programa SPSS 22 se analizaron estadísticamente la variable edad materna y se estimó las medidas de tendencia central (media, mediana y moda), luego la variable cuantitativa edad materna se transformó a una variable cualitativa, donde se halló la distribución porcentual según el grupo etareo. La variable edad gestacional se analizó su frecuencia de acuerdo a cada edad gestacional.

El índice de pulsatilidad de la arteria cerebral media se analizaron en el programa SPSS 22 por medio de una transformación natural logarítmica( $\log 10)$ de los valores del índice de pulsatilidad acuerdo a la edad gestacional, seguida de la regresión por estimación curvilínea modelo cuadrático para homogenizar los datos, pronosticando valores con un intervalo de predicción del 95\%, posteriormente se realiza una formula antilogarítmica a los resultados logarítmicos(valores relativos), obteniendo así valores representativos del índice de pulsatilidad en el percentil 5,50 y 95 para cada edad gestacional entre las
20 y 40 semanas de gestación. Para la elaboración de los cuadros y gráficos se utilizaron los programas Excel y SPSS para Windows 2013

\section{RESULTADOS}

Se seleccionaron a 1865 gestantes entre la 20 a 40 semanas que cumplían con los criterios de inclusión y realizaron un ultrasonido doppler en la Unidad de Medicina Fetal del INMP en el periodo de estudio establecido.

La edad materna más frecuente es entre los 20 a 34 años con un $66,2 \%$, seguido de las gestantes añosas que representaban el $24,9 \%$ y por último el $8,9 \%$ eran gestantes cuya edad cursaban entre los 13 a 19 años.(tabla 1).

Tabla 1. Distribución porcentual de la edad materna según grupo etareo.

\begin{tabular}{ccc}
\hline Edad materna & $\mathbf{N}$ & $\%$ \\
\hline 13 a 19 años & 166 & $8,9 \%$ \\
20 a 34 años & 1234 & $66,2 \%$ \\
35 años a Más & 465 & $24,9 \%$ \\
Total & 1865 & $100,0 \%$ \\
\hline
\end{tabular}

En la tabla 2 se observa los índices de pulsatilidad de la arteria cerebral media en el percentil 5,50 y 95 de acuerdo a cada edad gestacional. Se evidencia que el índice de pulsatilidad de la arteria cerebral media a las 20 semanas de gestación aumenta hasta alcanzar un pico máximo a las 28 hasta las 30 semanas de gestación y luego disminuye hasta finalizar la gestación.

En el percentil 5 el índice de pulsatilidad de la arteria cerebral media es de 1.00 a las 20 semanas de gestación, luego

Tabla 2. Valores de referencia del índice de pulsatilidad de la arteria cerebral media en fetos sanos de 20 a 40 semanas.

\begin{tabular}{ccccc}
\hline EG & N $^{\circ}$ GESTANTE & P5 & P50 & P95 \\
\hline 20 & 46 & 1.00 & 1.57 & 2.45 \\
21 & 38 & 1.06 & 1.65 & 2.58 \\
22 & 58 & 1.10 & 1.72 & 2.69 \\
23 & 76 & 1.15 & 1.79 & 2.80 \\
24 & 78 & 1.19 & 1.85 & 2.89 \\
\hline 25 & 69 & 1.22 & 1.90 & 2.97 \\
26 & 100 & 1.24 & 1.94 & 3.03 \\
27 & 115 & 1.26 & 1.97 & 3.08 \\
28 & 165 & 1.28 & 1.99 & 3.11 \\
\hline 29 & 144 & 1.28 & 2.00 & 3.12 \\
30 & 216 & 1.28 & 1.99 & 3.11 \\
31 & 186 & 1.27 & 1.98 & 3.09 \\
32 & 89 & 1.25 & 1.95 & 3.05 \\
33 & 61 & 1.23 & 1.91 & 2.99 \\
34 & 43 & 1.20 & 1.87 & 2.91 \\
\hline 35 & 84 & 1.16 & 1.81 & 2.83 \\
36 & 128 & 1.12 & 1.75 & 2.73 \\
\hline 37 & 56 & 1.07 & 1.67 & 2.61 \\
\hline 38 & 32 & 1.02 & 1.59 & 2.49 \\
\hline 39 & 50 & 0.97 & 1.51 & 2.36 \\
\hline 40 & 31 & 0.91 & 1.42 & 2.22 \\
\hline & & & & \\
\hline
\end{tabular}


asciende hasta alcanzar un pico máximo de IP de 1.28 en la semanas 28 , y posteriormente disminuye hasta el término de gestación con un valor de 0.91 en la semana 40 .

En el percentil 50 el índice de pulsatilidad de la arteria cerebral media es de 1.57 a las 20 semanas de gestación, luego asciende hasta alcanzar un pico máximo de IP de 2.00 a las 29 semanas, posteriormente disminuye hasta el término de gestación con un valor de IP de 1.42 en la semana 40.

En el percentil 95 el índice de pulsatilidad de la arteria cerebral media es de 2.45 a las 20 semanas de gestación, luego asciende hasta alcanzar un pico máximo de IP de 3.12 en la 29 semanas, posteriormente disminuye hasta el término de gestación con un valor de 2.22 en la semana 40.

En Grafico 1 se observa la curva normal el comportamiento del índice de pulsatilidad de la arteria cerebral media según la edad gestacional, con tendencia a formar una curva parabólica. Ello indica que los valores del IP de la ACM normalmente ascienden desde la semana 20 hasta alcanzar su pico máximo en la semana 29 , para luego descender hasta el terminar la gestación.

\section{CURVA NORMAL DEL ÍNDICE DE PULSATILIDAD DE} LA ARTERIA CEREBRAL MEDIA EN FETOS SANOS

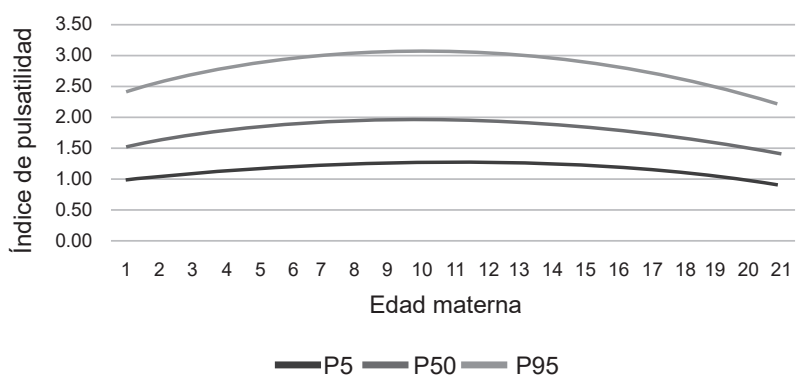

Gráfico 1. Curva normal del índice de pulsatilidad de la arteria cerebral media en fetos sanos.

\section{DISCUSIÓN}

Los resultados obtenidos por este estudio muestran que el índice de pulsatilidad de la arteria cerebral media en el percentil cinco aumenta hasta alcanzar un pico máximo de 1.28 a las 28 hasta 30 semanas de gestación, para luego disminuir hasta finalizar el embarazo. El estudio realizado por Arduini el pico máximo que alcanza el IP de ACM es 1.53 a las 27 hasta 29 semanas de gestación, de Baschat es de 1.45 a las 27 y 28 semanas de gestación, y de Tavares el IP de la ACM es 1.74 a las 26 hasta 28 semanas de gestación. Por lo tanto los valores obtenidos en el percentil 5 obtenidos por estos autores distan de los valores obtenidos por este estudio ${ }^{6-8}$.

En el estudio la curva normal del índice de pulsatilidad de la arteria cerebral media en relación a la edad gestacional forma un patrón parabólico al igual que los autores anteriormente mencionados, pero con valores distintos. Esta grafica evidencia que si los fetos de este estudio son evaluados con los parámetros de estos autores sus valores estarían por debajo del percentil cinco (<P5) ${ }^{9-11}$.

Tabla 3. Valores de referencia del índice de pulsatilidad de la arteria cerebral media en percentil cinco según varios autores.

\begin{tabular}{ccccc}
\hline EG & $\begin{array}{c}\text { P5 Arduini } \\
\text { Italia (1990) }\end{array}$ & $\begin{array}{c}\text { P5 Baschat } \\
\text { Alemania } \\
(2003)\end{array}$ & $\begin{array}{c}\text { P5 Tavares } \\
\text { Brasil(2013) }\end{array}$ & $\begin{array}{c}\text { P5 Del } \\
\text { Estudio Perú } \\
(2016)\end{array}$ \\
\hline 20 & 1.36 & & & 1.00 \\
21 & 1.4 & & & 1.06 \\
22 & 1.44 & & & 1.1 \\
\hline 23 & 1.47 & 1.37 & 1.65 & 1.15 \\
24 & 1.49 & 1.40 & 1.69 & 1.19 \\
\hline 25 & 1.51 & 1.43 & 1.72 & 1.22 \\
\hline 26 & 1.52 & 1.44 & 1.74 & 1.24 \\
\hline 27 & 1.53 & 1.45 & 1.74 & 1.26 \\
\hline 28 & 1.53 & 1.45 & 1.74 & 1.28 \\
\hline 29 & 1.53 & 1.44 & 1.73 & 1.28 \\
\hline 30 & 1.52 & 1.43 & 1.72 & 1.28 \\
\hline 31 & 1.51 & 1.41 & 1.70 & 1.27 \\
\hline 32 & 1.49 & 1.38 & 1.67 & 1.25 \\
\hline 33 & 1.46 & 1.34 & 1.63 & 1.23 \\
\hline 34 & 1.43 & 1.30 & 1.58 & 1.20 \\
\hline 35 & 1.4 & 1.25 & 1.51 & 1.16 \\
\hline 36 & 1.36 & 1.19 & 1.43 & 1.12 \\
\hline 37 & 1.32 & 1.13 & 1.36 & 1.07 \\
\hline 38 & 1.27 & 1.05 & 1.28 & 1.02 \\
\hline 39 & 1.08 & 0.98 & 1.19 & 0.97 \\
\hline 40 & 1.01 & 0.89 & 1.06 & 0.91 \\
\hline & & & & \\
\hline
\end{tabular}

Los valores del índice de pulsatilidad de la arteria cerebral media en el percentil 50 obtenidos por Arduini, Baschat y Tavares son similares al presente estudio. En la semana 28 de gestación el valor del índice de pulsatilidad en el percentil 50 según los resultados de Arduini es 1.99, Baschat es 2.05 , Tavares es 2.00 y el presente estudio es $1.99^{6-8}$.

La curva normal del índice de pulsatilidad de la arteria cerebral media en el percentil 50 obtenidos por Arduini, Baschat y Tavares forma un patrón parabólico a igual que el presente estudio. En este caso las curvas presentadas

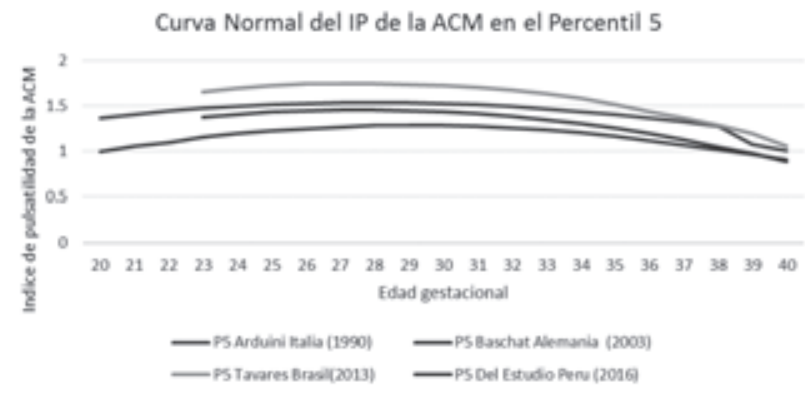

Grafico 2. Curva normal del índice de pulsatilidad de la arteria cerebral en el percentil cinco según varios autores. 
por los autores antes mencionados y del estudio son similares ${ }^{6-8}$.

Los resultados obtenidos por este estudio en comparación con los resultados de Arduini, Baschat y Tavares los valores del índice de pulsatilidad de la arteria cerebral media en el percentil 95 son diferentes. En la semana 28 de gestación el valor del índice de pulsatilidad en el percentil 95 según los resultados de Arduini es 2,46, Baschat es 2,66, Tavares es 2,30 y el presente estudio es $3,11^{6-8}$.

La curva normal del índice de pulsatilidad de la arteria cerebral media en el percentil 95 forma un patrón parabólico al igual que los autores Arduini, Baschat y Tavares $^{6-8}$, pero los valores de este estudio son más altos que los autores antes mencionados. Por lo tanto la curva es más elevada que el resto.

En conclusión se establecieron valores de referencia del índice de pulsatilidad de la arteria cerebral media en el percentil 5,50 y 95.Los valores del índice de pulsatilidad de la ACM entre el percentil 5 y 95 demuestra tener un valor clínico en la detección de fetos en condiciones óptimas.

La curva normal del índice de pulsatilidad de la arteria cerebral media en el percentil 5,50 y 95 de fetos sanos en relación a la edad gestacional sigue un patrón parabólico. Se evidencia que el índice de pulsatilidad asciende desde la semana 20 hasta alcanzar un pico máximo en la semana 28 hasta la semana 30 y luego disminuye hasta el término de la gestación.

Se recomienda que la tabla de valores del índice de pulsatilidad de la arteria cerebral media construidos sea considerada como parámetros referenciales para identificar y monitorear el bienestar fetal en la Institución Nacional Materno Perinatal y otras instituciones del Perú. Se recomienda que la presente estudio sea considerado para realizar otras investigaciones.

Financiamiento: autofinanciado.

Conflicto de interés: los autores declaran no tener algún conflicto de interés.

\section{REFERENCIAS BIBLIOGRÁFICAS}

1. Hemlata D, Hemant K, Anupama D. Middle Cerebral Artery Doppler Indices Better Predictor for Fetal Outcome in IUGR. The Journal of Obstetrics and Gynecology of India 2011; pg $166-171$.

2. Kuber R, Randhawa S, Khaladkar S, Patil A. Doppler study of middle cerebral artery and umbilical artery in biometrically suspected intra uterine growth restricted pregnancies. Int J Rev Med Sci. 2016 ;4(2):403-414

3. Borges A, Mara T, Godoy T y Cols.Assessment of ultrasound and Doppler parameters in the third trimester of pregnancy as predictorsof adverse perinatal outcome in unselected pregnancies. Ginekologia Polska (2016); 87( 7): 510-515

4. Cruz-Martinez R, Tenorio V, Padilla N, Crispi F, Figueras F, Gratacos E. Risk of ultrasound-detected neonatal brain abnormalities in intrauterine growth-restricted fetuses born between 28 and 34 weeks' gestation: relationship with gestational age at birth and fetal Doppler parameters. Ultrasound Obstet Gynecol 2015; 46: 452-459

5. Medina N, Figueroa H, Hernández E. Valores de referencia del índice de pulsatilidad y de la velocidad máxima en la arteriacerebral media fetal durante el embarazo normal. Ginecol Obstet Mex 2006;74 :376-82 .

6. Arduini D, Rizzo G.Normal values of pulsality index from fetal vessel :A cross-sectional study on 1566 healthy fetuses. J PerinatMed 1990; 18: 165-172

7. Baschat y Gembruch.The cerebroplacental Doppler ratio revisited. Ultrasound Obstet Gynecol 2003; 21: 124-127

8. Tavares NM, Ferreira SG, Bennini JR, Marussi EF, Barini $\mathrm{R}$, Peralta CF. Longitudinal reference intervals of maternalfetal Doppler parameters. Rev Bras Ginecol Obstet. 2013; 35(1):33-8.

9. Gratacós E, Figueras F, Hernández E, Puerto B., Doppler en medicina fetal: Técnicas y aplicación clínica. Madrid: Editorial Médica Panamericana; 2012

10. Morales $\mathrm{J}$ y col.Doppler reference values of the fetal vertebral and middle cerebral arteries, at 19-41 weeks gestation. Rev Bras Ginecol Obstet. 2013; 35(1):33-8.

11. Tarzamni M, Nezami N, Samani F, Vahedinia S, Tarzamni M.Doppler Waveform Indices of Fetal Middle Cerebral Artery in Normal 20 to 40 Weeks Pregnancies. Arch Iranian Med 2009; 12 (1): $29-34$.

\section{Correspondencia:}

Dirección: Av. Javier Prado Este 175, Piso 3. Of. 304 -San IsidroLima.

Teléfono: (51 1) 447-8056 Celular: (51 1) 984-840625

E-mail: antoniolimay@hotmail.com

\section{ORCID iDs}

Oscar Antonio Limav Ríos

Jaime Ingar Pinedo

Erasmo Huertas Tacchino

Walter Castillo Urquiaga

Walter Ventura Laveriano https://orcid.ora/0000-0001-6012-3705

https://orcid.org/0000-0001-6472-7899

https://orcid.org/0000-0002-9851-8419

hittps://orcid.org/0000-0002-1054-7398

idi) htps://orcid.org/0000-0002-2888-1394 